\title{
Alterações nas propriedades físicas de substratos para cultivo de tomate cereja, em função de sua reutilização
}

\section{Carolina Fernandes; José Eduardo Corá; Leila T Braz}

UNESP, Via de acesso Prof. Paulo Donato Castellane, s/n, 14884-900 Jaboticabal-SP; E-mail: carol@fcav.unesp.br; cora@fcav.unesp.br; leilatb@fcav.unesp.br

\section{RESUMO}

O objetivo deste trabalho foi avaliar as alterações nas propriedades físicas de substratos em função de sua reutilização para o cultivo do tomateiro do grupo cereja, cultivar Sindy. O delineamento experimental adotado foi em parcelas subdivididas com os sete substratos (nas parcelas) e três épocas de caracterização física dos substratos (nas subparcelas). As parcelas foram agrupadas em blocos casualizados, com quatro repetições. Os sete substratos resultaram da combinação de diferentes proporções volumétricas de três componentes: areia, bagaço de cana-de-açúcar e casca de amendoim. As propriedades físicas dos substratos avaliadas foram submetidas à análise de variância, sendo as médias comparadas pelo teste de Tukey a $5 \%$ de probabilidade. A reutilização do substrato composto por partes iguais dos três componentes promoveu aumento da densidade seca e do volume de água facilmente disponível, e redução da porosidade total, do espaço de aeração e do volume de água remanescente.

Palavras-chave: Lycopersicon esculentum, areia, bagaço de canade-açúcar, casca de amendoim, cultivo sem solo.

\begin{abstract}
Effects of the reutilization of substrates on its physical properties on growing cherry tomato

The objective of this work was to evaluate the effects of the reutilization on the physical properties of different types of substrate used to grow cherry tomato, at Jaboticabal, São Paulo State, Brazil (2114'05" S, 48 $\left.{ }^{\circ} 17^{\prime} 09^{\prime \prime} \mathrm{W}\right)$. We used the split-plot experimental design with complete randomized blocks and four replications. In the plots were placed seven types of substrates, and in the subplots, three periods of physical characterization of the substrates. The seven substrates were prepared with different combinations of three materials: sand, crushed sugarcane and peanut bark. The physical properties evaluated were submitted to variance analysis and the averages were compared by the Tukey test, at $5 \%$ probability level. The reutilization of the substrate prepared with equal parts of the three components promoted the increase of bulk density and easily available water content, and the decrease of total porosity, aeration space, and remaining water content.
\end{abstract}

Keywords: Lycopersicon esculentum, sand, crushed sugarcane, peanut bark, soilless growth.

\section{(Recebido para publicação em 05 de abril de 2005; aceito em 10 de fevereiro de 2006)}

\begin{abstract}
A crescente demanda por hortaliças de alta qualidade e ofertadas durante $\mathrm{o}$ ano todo tem contribuído para o investimento em novos sistemas de cultivo, que permitam produção adaptada a diferentes regiões e condições adversas do ambiente (Carrijo et al., 2004). No Brasil, nota-se um crescente interesse dos produtores pelo cultivo de hortaliças em substratos.

A avaliação da qualidade de um substrato baseia-se na sua caracterização física e química. Dentre as propriedades físicas, destacam-se a distribuição do tamanho das partículas, a densidade e a curva de retenção de água. A composição granulométrica, que representa a distribuição do tamanho das partículas, tem influência determinante no volume de ar e água retidos pelo substrato (Ansorena, 1994). A densidade, que expressa a relação entre a massa e o volume ocupado pelas partículas que formam o substrato, pode se alterar
\end{abstract}

em função do manejo, conseqüentemente, as demais propriedades físicas do substrato também podem ser modificadas. A determinação da curva de retenção de água para um substrato é importante, porque fornece dados sobre o volume de água em determinadas tensões (De Boodt \& Verdonck, 1972). A partir desses valores, pode-se calcular porosidade total, espaço de aeração, água facilmente disponível, água tamponante e água remanescente.

A reutilização de substratos caracteriza-se como uma possibilidade de reduzir o custo de produção, uma vez que dispensa a aquisição de novos substratos. Além disso, pode-se conseguir um menor impacto ambiental, uma vez que a reutilização possibilita a redução do volume de substrato descartado após o cultivo.

Algumas pesquisas têm evidenciado resultados econômicos positivos com a reutilização de substratos por dois ou mais cultivos consecutivos, sem reduzir a produção e a qualidade de hortaliças (Baevre, 1981; Baevre \& Guttormsen, 1984; Verlodt et al., 1985; Andriolo et al., 1999; Celikel \& Caglar, 1999; Reis et al., 2001). Entretanto, deve-se avaliar as alterações nas propriedades físicas dos substratos reutilizados, para que o manejo da irrigação, nos cultivos sucessivos, possa ser modificado em função das propriedades físicas dos substratos reutilizados.

Baevre \& Guttormsen, (1984) observaram que a reutilização do substrato não alterou significativamente a porosidade total, mas reduziu o volume de poros maiores e aumentou o volume de poros menores. A reutilização do substrato por cinco anos proporcionou aumento da densidade, diminuição da porosidade total, aumento do conteúdo de água e diminuição do espaço de aeração (Verlodt et al., 1985). Os autores relacionaram essas alterações físi- 
cas com o aumento linear da decomposição do substrato em função dos anos de cultivo.

A decomposição de substratos orgânicos promove a redução no tamanho das partículas, conseqüentemente, no tamanho dos poros formados pelas mesmas (Ansorena, 1994). De acordo com Andriolo et al. (1999), a vida útil de um substrato orgânico é determinada, principalmente, pela velocidade das reações de decomposição, que modificam a granulometria do material e, conseqüentemente, a proporção entre as fases sólida, líquida e gasosa.

Vários são os materiais utilizados como substratos para plantas, tais como, turfa, areia, isopor, espuma fenólica, argila expandida, perlita, vermiculita, casca de arroz, casca de pínus, fibra da casca de coco, serragem, entre outros. Entretanto, Carrijo et al. (2004), ressaltam que a caracterização de produtos encontrados nas diferentes regiões do país é fundamental para reduzir o custo de produção de hortaliças em substratos.

Devido às atividades agrícolas desenvolvidas na região de Jaboticabal, bagaço de cana-de-açúcar e casca de amendoim são resíduos disponíveis em grande quantidade, apresentando, portanto, potencial para serem utilizados na composição de substratos.

O objetivo deste trabalho foi avaliar as alterações nas propriedades físicas de sete substratos, compostos por areia, bagaço de cana-de-açúcar e casca de amendoim, em função de sua reutilização, para o cultivo do tomateiro do grupo cereja, cultivar Sindy.

\section{MATERIAL E MÉTODOS}

Foram realizados dois cultivos do tomateiro do grupo cereja, cultivar Sindy, em casa de vegetação, no Setor de Olericultura e Plantas AromáticoMedicinais, na UNESP em Jaboticabal. O clima, segundo a classificação de Köppen, é do tipo Aw com transição para Cwa. A casa de vegetação foi construída em estrutura metálica, do tipo teto em arco, com $3 \mathrm{~m}$ de pé-direito, 30 $\mathrm{m}$ de comprimento e $8 \mathrm{~m}$ de largura, coberta com filme de polietileno transparente, aditivado contra raios ultravioleta, com $150 \mathrm{~mm}$ de espessura,
Tabela 1. Distribuição do tamanho de partículas dos componentes e dos substratos utilizados no cultivo do tomateiro do grupo cereja, cultivar Sindy, em casa de vegetação. Jaboticabal, UNESP, 2004.

\begin{tabular}{|c|c|c|c|c|c|c|c|}
\hline \multirow{3}{*}{ Componentes/Substratos } & \multicolumn{7}{|c|}{ Diâmetro das partículas (mm) } \\
\hline & $<0,125$ & $0,125-0,25$ & $0,25-0,5$ & $0,5-1$ & $1-2$ & $2-4$ & $>4$ \\
\hline & \multicolumn{7}{|c|}{$\%$ em peso } \\
\hline Bagaço de cana-de-açúcar (BC) & 3 & 4 & 12 & 24 & 25 & 22 & 10 \\
\hline Casca de amendoim (CA) & 2 & 6 & 7 & 12 & 23 & 49 & 1 \\
\hline Areia $(A)$ & 4 & 32 & 49 & 11 & 2 & 2 & 0 \\
\hline $2 / 3 A+1 / 3 B C$ & 4 & 31 & 48 & 11 & 3 & 2 & 1 \\
\hline $2 / 3 A+1 / 3 C A$ & 2 & 27 & 48 & 12 & 5 & 6 & 0 \\
\hline $2 / 3 A+1 / 6 B C+1 / 6 C A$ & 3 & 30 & 48 & 11 & 4 & 4 & 0 \\
\hline $1 / 2 A+1 / 2 B C$ & 3 & 29 & 49 & 12 & 3 & 3 & 1 \\
\hline $1 / 2 A+1 / 2 C A$ & 2 & 29 & 46 & 11 & 5 & 7 & 0 \\
\hline $1 / 3 A+1 / 3 B C+1 / 3 C A$ & 4 & 26 & 42 & 13 & 6 & 8 & 1 \\
\hline
\end{tabular}

e as laterais protegidas com telas de polipropileno preto com $50 \%$ de sombreamento. A temperatura e a umidade relativa do ar, no interior da casa de vegetação, foram obtidas por meio de um termohigrógrafo com registro contínuo dos valores. $\mathrm{O}$ aparelho foi instalado em abrigo de madeira, a 1,0 $m$ de altura, no centro da casa de vegetação. Durante o primeiro cultivo, as médias mensais para a temperatura máxima diária, nos meses de fevereiro, março, abril e maio foram, respectivamente, $39,36,33$ e $29^{\circ} \mathrm{C}$; para a temperatura mínima diária, 21, 18, 15 e 11 ${ }^{\circ} \mathrm{C}$; para a umidade relativa do ar máxima diária, 93, 95, 97 e 99\%; e para a umidade relativa do ar mínima diária, 37, 38, 39 e 40\%. Durante o segundo cultivo, as médias mensais para a temperatura máxima diária, nos meses de julho, agosto, setembro, outubro e novembro foram, respectivamente, 33,33 , 35,36 e $38{ }^{\circ} \mathrm{C}$; para a temperatura mínima diária, $11,11,13,14$ e $16^{\circ} \mathrm{C}$; para a umidade relativa do ar máxima diária, 98, 98, 96, 95 e 94\%; e para a umidade relativa do ar mínima diária, 40, 40, 39, 38 e $37 \%$.

O delineamento experimental adotado foi em parcelas subdivididas com sete substratos (nas parcelas) e três épocas de caracterização física dos substratos (nas subparcelas). As parcelas foram agrupadas em blocos casualizados, com quatro repetições. A unidade experimental foi representada por dois vasos, contendo uma planta por vaso. Os vasos utilizados foram de plástico de cor marrom, com capacidade para $5 \mathrm{~L}$, com $25 \mathrm{~cm}$ de diâmetro de boca, $17 \mathrm{~cm}$ de diâmetro de fundo, 18 $\mathrm{cm}$ de altura e 8 furos na base.

Os substratos resultaram da combinação de diferentes proporções volumétricas de areia (A), bagaço de cana-de-açúcar (BC) e casca de amendoim (CA). A areia utilizada foi a conhecida comercialmente como areia média. $\mathrm{O}$ bagaço utilizado foi o resíduo fibroso resultante da extração do caldo dos colmos da cana-de-açúcar. A casca de amendoim utilizada foi moída e passada em peneira com abertura de $6 \mathrm{~mm}$ $\mathrm{x} 18 \mathrm{~mm}$. Os substratos testados foram: $\mathrm{S}_{1}=\mathrm{A} ; \mathrm{S}_{2}=2 / 3 \mathrm{~A}+1 / 3 \mathrm{BC} ; \mathrm{S}_{3}=2 / 3 \mathrm{~A}$ $+1 / 3 \mathrm{CA} ; \mathrm{S}_{4}=2 / 3 \mathrm{~A}+1 / 6 \mathrm{BC}+1 / 6$ $\mathrm{CA} ; \mathrm{S}_{5}=1 / 2 \mathrm{~A}+1 / 2 \mathrm{BC} ; \mathrm{S}_{6}=1 / 2 \mathrm{~A}+1 /$ $2 \mathrm{CA} \mathrm{S}_{7}=1 / 3 \mathrm{~A}+1 / 3 \mathrm{BC}+1 / 3 \mathrm{CA}$.

Para cada componente dos substratos, determinou-se a densidade seca (Ds), segundo Hoffmann (1970), citado por Backes (1988) e a distribuição do tamanho de partículas (granulometria), utilizando-se peneiras de 4,$0 ; 2,0 ; 1,0 ; 0,5 ; 0,25$ e $0,125 \mathrm{~mm}$ de abertura. Os valores da densidade seca foram: $1.691 \mathrm{~kg} \mathrm{~m}^{-3}$ para a areia, $58 \mathrm{~kg} \mathrm{~m}^{-3}$ para o bagaço de cana-de-açúcar e $186 \mathrm{~kg} \mathrm{~m}^{-3}$ para a casca de amendoim. A distribuição do tamanho de partículas dos componentes dos substratos encontra-se na Tabela 1.

Posteriormente, os componentes foram misturados nas diferentes combinações e a granulometria dos substratos (Tabela 1) foi determinada como descrito anteriormente.

Procedeu-se, então, ao preenchimento dos vasos com os diferentes 


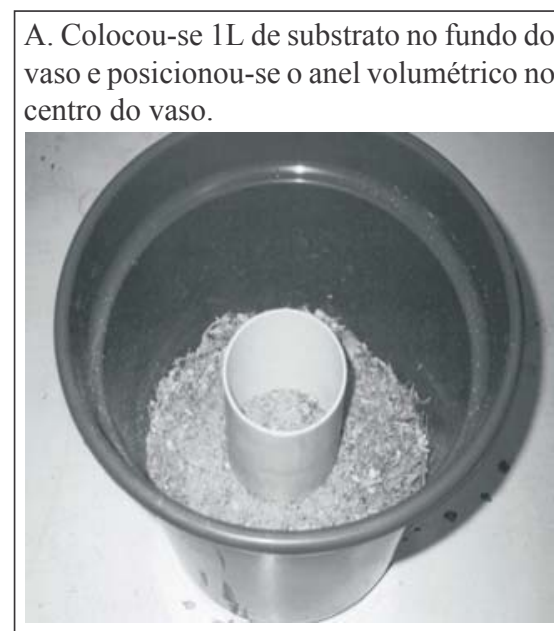

C. Prosseguiu-se com o preenchimento do vaso e do anel volumétrico.

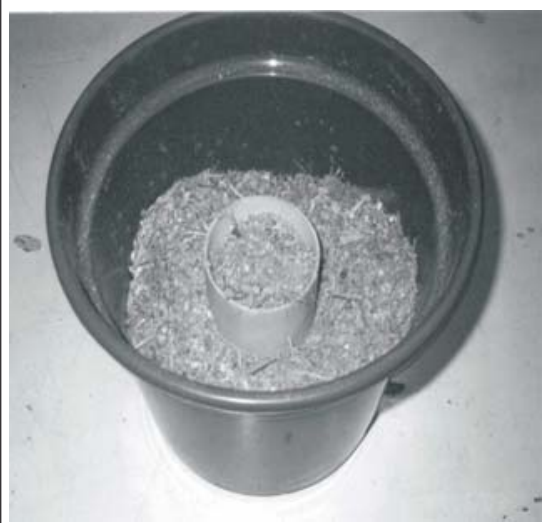

Figura 1. Preenchimento dos vasos.

substratos. No centro de cada vaso, foi colocado um anel volumétrico de PVC com aproximadamente $285 \mathrm{~cm}^{3}(7,2 \mathrm{~cm}$ de diâmetro e $7,0 \mathrm{~cm}$ de altura), conforme apresentado na Figura 1, com o objetivo de determinar densidade seca, porosidade total (PT), espaço de aeração (EA), água facilmente disponível (AFD), água tamponante (AT) e água remanescente (AR), segundo De Boodt \& Verdonck (1972), nas três épocas de caracterização física dos substratos.

As épocas de caracterização física dos substratos foram: $\mathrm{C}_{0}=$ antes do primeiro cultivo, $\mathrm{C}_{1}$ = após o primeiro cultivo e $\mathrm{C}_{2}=$ após o segundo cultivo.

Antes do transplantio das mudas para o primeiro cultivo, os vasos já preenchidos com os diferentes substratos foram alocados na casa de vegetação e irrigados, durante uma semana, a fim de promover a acomodação natural das partículas dos substratos e uniformizar a umidade dos substratos dentro do vaso. Depois desse período, realizou-se a pri-
B. Adicionou-se substrato lentamente para preencher o anel volumétrico e o vaso.

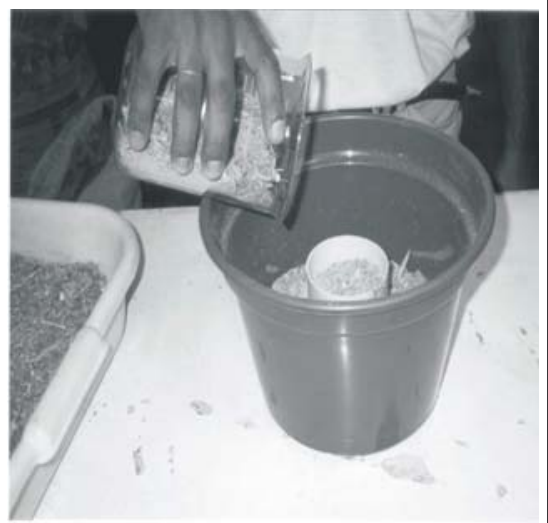

D. Vaso e anel volumétrico totalmente preenchidos.

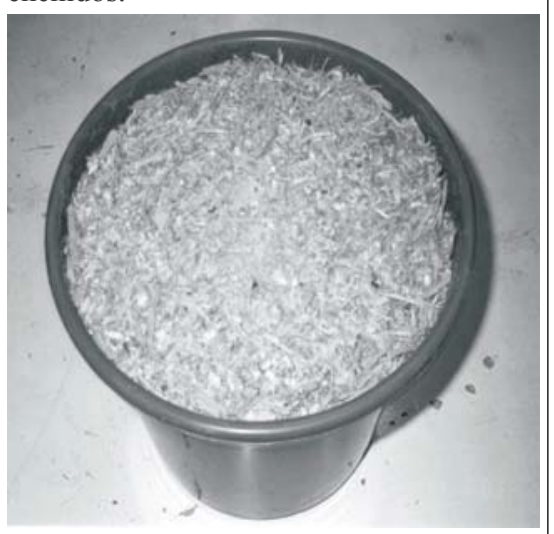

meira determinação das propriedades físicas dos substratos $\left(\mathrm{C}_{0}\right)$. Porém, como a casa de vegetação não comportava o número de vasos necessários para realizar essa caracterização, reproduziu-se essa fase em laboratório. Para tanto, foram utilizados 35 vasos (cinco replicações de cada um dos sete substratos), que foram preenchidos com os diferentes substratos, conforme descrito anteriormente. Diariamente, os 35 vasos receberam, manualmente, o mesmo volume de água que os vasos alocados na casa de vegetação receberam, via irrigação. Após uma semana, os 35 vasos foram desmontados e os anéis volumétricos foram cuidadosamente retirados dos vasos, conforme apresentado na Figura 2, para a determinação das propriedades físicas dos substratos, antes do primeiro cultivo $\left(\mathrm{C}_{0}\right)$.

Para o primeiro cultivo, todos os vasos foram preenchidos com os diferentes substratos até então não utilizados. Após o primeiro cultivo, uma me- tade dos vasos foi utilizada para posterior caracterização física dos substratos. A outra metade dos vasos, utilizada no primeiro cultivo, foi utilizada no segundo cultivo. Para isso, os substratos não foram removidos dos vasos. Somente a parte aérea da planta, proveniente do primeiro cultivo, foi retirada, cortandose a haste próximo à superfície do substrato, deixando-se o sistema radicular. Para a determinação das propriedades físicas dos substratos, após o primeiro $\left(\mathrm{C}_{1}\right)$ e segundo cultivo $\left(\mathrm{C}_{2}\right)$, os vasos foram desmontados, e os anéis volumétricos foram cuidadosamente retirados dos vasos, conforme apresentado na Figura 2.

As variáveis avaliadas foram submetidas à análise de variância, e as médias comparadas pelo teste de Tukey a 5\% de probabilidade.

\section{RESULTADOS E DISCUSSÃO}

Os resultados demonstraram que houve diferenças significativas nas propriedades físicas dos sete substratos, exceto para o volume de água tamponante. Entretanto, somente o substrato $\mathrm{S}_{7}(1 / 3 \mathrm{~A}+1 / 3 \mathrm{BC}+1 / 3 \mathrm{CA})$ apresentou alterações nas propriedades físicas em função da sua utilização nos dois cultivos (Tabela 2).

Os valores da densidade seca dos substratos (Ds) decresceram com a diminuição do volume de areia e o aumento de bagaço de cana-de-açúcar (BC) e/ ou de casca de amendoim (CA) (Tabela $2)$, porque os valores de densidade seca dos componentes BC $\left(58 \mathrm{~kg} \mathrm{~m}^{-3}\right)$ e CA $\left(186 \mathrm{~kg} \mathrm{~m}^{-3}\right)$ foram menores, quando comparados com a densidade seca da areia $\left(1.691 \mathrm{~kg} \mathrm{~m}^{-3}\right)$.

Os valores da porosidade total dos substratos (PT) aumentaram com a diminuição do volume de areia e o aumento de bagaço de cana-de-açúcar (BC) e/ ou de casca de amendoim (CA) (Tabela 2), possivelmente em decorrência da redução na quantidade de partículas menores, provenientes do componente areia, e aumento na quantidade de partículas maiores, provenientes dos componentes BC e CA. A areia apresentou predominância de partículas com diâmetro entre $0,125 \mathrm{~mm}$ e $0,50 \mathrm{~mm}$, o bagaço de cana-de-açúcar com diâmetro 
maior que $0,25 \mathrm{~mm}$ e a casca de amendoim com diâmetro entre 1,00 e 4,00 mm (Tabela 1).

O maior valor do espaço de aeração (EA) e o menor valor da água facilmente disponível (AFD) foram observados no substrato com $1 / 3 \mathrm{~A}+1 / 3 \mathrm{BC}+1 / 3$ CA (Tabela 2), provavelmente, por causa da sua granulometria. Esse substrato apresentou a maior porcentagem de partículas com diâmetro maior que $1,0 \mathrm{~mm}$ e a menor porcentagem de partículas com diâmetro entre $0,25 \mathrm{~mm}$ e $1,0 \mathrm{~mm}$ (Tabela 1). De acordo com Richards et al. (1986) e Abad et al. (2004), partículas com diâmetro maior que $1,0 \mathrm{~mm}$ formam poros responsáveis pelo armazenamento de ar e partículas com diâmetro entre $0,25 \mathrm{~mm}$ e $1,0 \mathrm{~mm}$ formam poros responsáveis pelo armazenamento de água facilmente disponível às plantas.

Comparando-se as épocas de caracterização física do substrato $\mathrm{S}_{7}$, antes $\left(\mathrm{C}_{0}\right)$ e depois do primeiro cultivo $\left(\mathrm{C}_{1}\right)$, observou-se que o valor da densidade seca (Ds) aumentou e proporcionou a redução da porosidade total (PT) (Tabela 2). Os volumes do espaço de aeração (EA) e da água remanescente (AR) diminuíram, e o volume da água facilmente disponível (AFD) aumentou. Assim, durante o primeiro cultivo, constatou-se a redução do volume total de poros e a alteração no tamanho dos poros do substrato $\mathrm{S}_{7}$. Esses resultados corroboram com aqueles obtidos por Verlodt et al. (1985), que observaram aumento da densidade, diminuição da porosidade total, aumento do volume de água e diminuição do volume de ar no substrato reutilizado por cinco anos.

Comparando-se as épocas de caracterização física do substrato $\mathrm{S}_{7}$, antes $\left(C_{1}\right)$ e depois do segundo cultivo $\left(C_{2}\right)$, observou-se redução do EA e aumento da AFD (Tabela 2). Assim, durante o segundo cultivo, constatou-se apenas a alteração no tamanho dos poros do substrato $\mathrm{S}_{7}$. Esses resultados corroboram com aqueles obtidos por Baevre \& Guttormsen (1984), que observaram redução do volume de poros maiores e aumento do volume de poros menores, sem, entretanto, alterar a porosidade total do substrato reutilizado.

A redução do volume total de poros do substrato $\mathrm{S}_{7}$, observada durante o

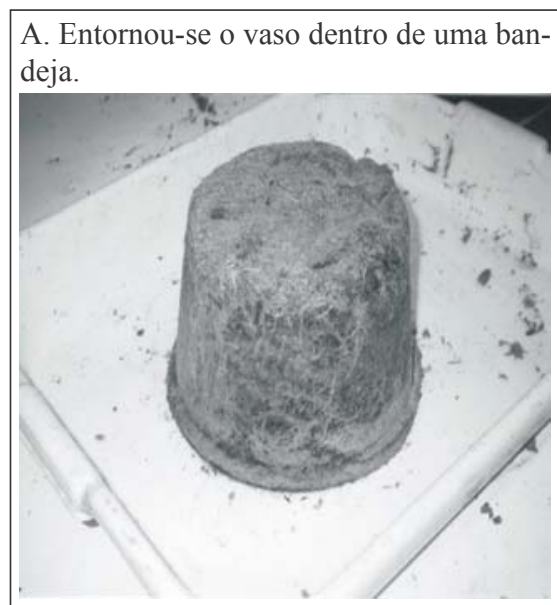

C. Nivelou-se as duas extremidades do anel volumétrico.

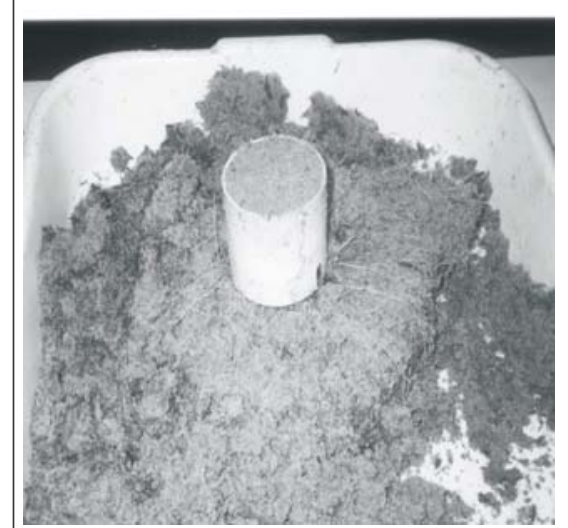

Figura 2. Retirada dos anéis volumétricos.

primeiro cultivo, pode ter sido provocada pela irrigação, que promoveu a movimentação das partículas do substrato, fazendo com que as menores ocupassem os espaços formados entre as maiores. Além disso, o desenvolvimento do sistema radicular do tomateiro também pode ter promovido o arrastamento das partículas do substrato. Essa movimentação e acomodação das partículas promoveram o adensamento do substrato e, conseqüentemente, a redução da porosidade total. Porém, durante o segundo cultivo, não se observou redução do volume total de poros, possivelmente porque a relação entre massa e volume do substrato $\mathrm{S}_{7}$ acondicionado no vaso estabilizou-se durante o primeiro cultivo.

A alteração no tamanho dos poros do substrato $\mathrm{S}_{7}$, observada durante os dois cultivos, pode ter sido provocada pela decomposição do bagaço de canade-açúcar e da casca de amendoim, reduzindo o tamanho das partículas des-
B. Retirou-se o substrato até encontrar o anel volumétrico.

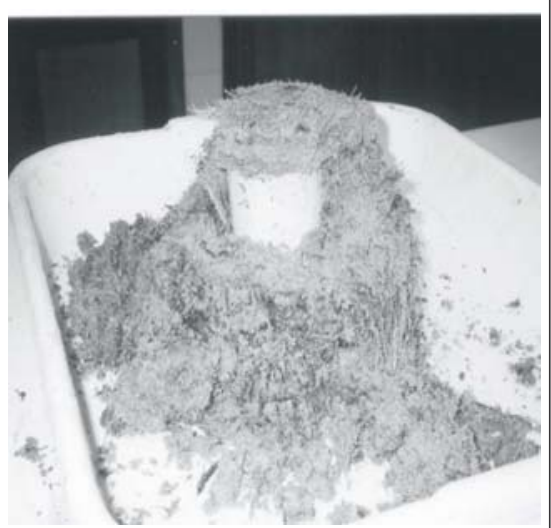

D. Anel volumétrico pronto para a caracterização física.

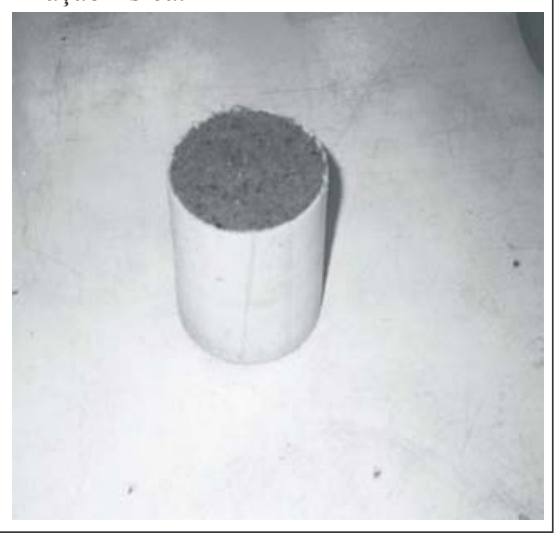

ses componentes. Assim, as partículas maiores, que proporcionavam a formação de poros maiores, responsáveis pelo armazenamento de ar, reduziram de tamanho e passaram a formar poros menores, responsáveis pelo armazenamento de água.

A redução do volume de água remanescente (AR) do substrato $\mathrm{S}_{7}$, observada durante o primeiro cultivo, pode ter sido provocada pelo desenvolvimento de raízes finas entre as partículas do substrato, promovendo o aumento do diâmetro dos poros. Assim, reduziu o volume de poros responsáveis pelo armazenamento de água remanescente e aumentou o volume de poros responsáveis pelo armazenamento de água facilmente disponível.

Ao contrário do substrato $\mathrm{S}_{7}(1 / 3 \mathrm{~A}$ $+1 / 3 \mathrm{BC}+1 / 3 \mathrm{CA})$, os demais substratos não apresentaram alterações nas propriedades físicas em função da utilização nos dois cultivos (Tabela 2). Possivelmente, por possuir maior volu- 
Tabela 2. Densidade seca (Ds), porosidade total (PT), espaço de aeração (EA), água facilmente disponível (AFD), água tamponante (AT) e água remanescente (AR), em três épocas de caracterização, dos sete substratos utilizados no cultivo do tomateiro do grupo cereja, cultivar Sindy, em casa de vegetação. Jaboticabal, UNESP, 2004.

\begin{tabular}{|c|c|c|c|c|c|c|c|c|}
\hline \multirow{2}{*}{\multicolumn{2}{|c|}{$\begin{array}{c}\text { Épocas de } \\
\text { caracterização }\end{array}$}} & \multicolumn{7}{|c|}{ Substratos $^{2}$} \\
\hline & & $\mathbf{S}_{1}$ & $\mathrm{~S}_{2}$ & $\mathrm{~S}_{3}$ & $\mathrm{~S}_{4}$ & $\mathbf{S}_{5}$ & $\mathbf{S}_{6}$ & $S_{7}$ \\
\hline & $\mathrm{C}_{0}$ & $1631 \mathrm{~A}$ & $1343 \mathrm{~B}$ & $1313 \mathrm{~B}$ & $1387 \mathrm{~B}$ & $1051 \mathrm{C}$ & $1103 \mathrm{C}$ & $680 \mathrm{Db}$ \\
\hline \multirow[t]{3}{*}{ Ds $\left(\mathrm{kg} \mathrm{m}^{-3}\right)$} & $C_{1}$ & $1604 \mathrm{~A}$ & $1414 \mathrm{~B}$ & $1379 \mathrm{~B}$ & $1353 \mathrm{~B}$ & $1142 \mathrm{C}$ & $1073 \mathrm{C}$ & $791 \mathrm{Da}$ \\
\hline & $\mathrm{C}_{2}$ & $1598 \mathrm{~A}$ & $1372 \mathrm{~B}$ & $1340 \mathrm{~B}$ & $1309 \mathrm{~B}$ & $1131 \mathrm{C}$ & $1049 \mathrm{C}$ & $793 \mathrm{Da}$ \\
\hline & $\mathrm{C}_{0}$ & $32 \mathrm{D}$ & $44 \mathrm{C}$ & $41 \mathrm{C}$ & $41 \mathrm{C}$ & $53 \mathrm{~B}$ & $49 \mathrm{~B}$ & $64 \mathrm{Aa}$ \\
\hline \multirow[t]{3}{*}{ PT (\%) } & $C_{1}$ & $34 \mathrm{D}$ & $43 \mathrm{C}$ & $42 \mathrm{C}$ & $44 \mathrm{C}$ & $53 \mathrm{~B}$ & $52 \mathrm{~B}$ & $59 \mathrm{Ab}$ \\
\hline & $\mathrm{C}_{2}$ & $34 \mathrm{D}$ & $40 \mathrm{C}$ & $42 \mathrm{C}$ & $42 \mathrm{C}$ & $51 \mathrm{~B}$ & $53 \mathrm{~B}$ & $58 \mathrm{Ab}$ \\
\hline & $\mathrm{C}_{0}$ & $1 \mathrm{D}$ & $4 \mathrm{D}$ & $4 \mathrm{D}$ & $3 \mathrm{D}$ & $10 \mathrm{C}$ & $10 \mathrm{~B}$ & $28 \mathrm{Aa}$ \\
\hline \multirow[t]{3}{*}{ EA (\%) } & $\mathrm{C}_{1}$ & $2 \mathrm{D}$ & $3 \mathrm{D}$ & $4 \mathrm{D}$ & $4 \mathrm{D}$ & $10 \mathrm{C}$ & $12 \mathrm{~B}$ & $23 \mathrm{Ab}$ \\
\hline & $\mathrm{C}_{2}$ & $2 \mathrm{D}$ & $2 \mathrm{D}$ & $4 \mathrm{D}$ & $4 \mathrm{D}$ & $8 \mathrm{C}$ & $10 \mathrm{~B}$ & $19 \mathrm{Ac}$ \\
\hline & $\mathrm{C}_{0}$ & $24 \mathrm{~A}$ & $27 \mathrm{~A}$ & $24 \mathrm{~A}$ & $25 \mathrm{~A}$ & $24 \mathrm{~A}$ & $20 \mathrm{~B}$ & $12 \mathrm{Cc}$ \\
\hline \multirow[t]{3}{*}{ AFD (\%) } & $\mathrm{C}_{1}$ & $23 \mathrm{~A}$ & $25 \mathrm{~A}$ & $23 \mathrm{~A}$ & $25 \mathrm{~A}$ & $23 \mathrm{~A}$ & $20 \mathrm{~B}$ & $16 \mathrm{Cb}$ \\
\hline & $\mathrm{C}_{2}$ & $22 \mathrm{~A}$ & $23 \mathrm{~A}$ & $24 \mathrm{~A}$ & $23 \mathrm{~A}$ & $24 \mathrm{~A}$ & $23 \mathrm{~A}$ & $20 \mathrm{Ba}$ \\
\hline & $\mathrm{C}_{0}$ & 3 & 2 & 3 & 2 & 4 & 2 & 1 \\
\hline \multirow[t]{3}{*}{ AT $(\%)$} & $\mathrm{C}_{1}$ & 3 & 2 & 3 & 3 & 3 & 3 & 1 \\
\hline & $\mathrm{C}_{2}$ & 4 & 4 & 3 & 4 & 4 & 5 & 3 \\
\hline & $\mathrm{C}_{0}$ & $4 \mathrm{E}$ & 11D & $10 \mathrm{D}$ & 11D & $15 \mathrm{C}$ & $17 \mathrm{~B}$ & $23 \mathrm{Aa}$ \\
\hline \multirow[t]{2}{*}{ AR $(\%)$} & $C_{1}$ & $6 \mathrm{C}$ & $13 \mathrm{~B}$ & $12 \mathrm{~B}$ & $12 \mathrm{~B}$ & $17 \mathrm{~A}$ & $17 \mathrm{~A}$ & $19 \mathrm{Ab}$ \\
\hline & $\mathrm{C}_{2}$ & $6 \mathrm{C}$ & 11B & 11B & 11B & $15 \mathrm{~A}$ & $15 \mathrm{~A}$ & $16 \mathrm{Ab}$ \\
\hline
\end{tabular}

${ }^{1} \mathrm{C}_{0}=$ antes do primeiro cultivo; $\mathrm{C}_{1}=$ após o primeiro cultivo; $\mathrm{C}_{2}=$ após o segundo cultivo; ${ }^{2} \mathrm{~S}_{1}=\mathrm{A} ; \mathrm{S}_{2}=2 / 3 \mathrm{~A}+1 / 3 \mathrm{BC} ; \mathrm{S}_{3}=2 / 3 \mathrm{~A}+1 / 3 \mathrm{CA} ; \mathrm{S}_{4}=2 / 3 \mathrm{~A}+1 / 6 \mathrm{BC}+1 / 6 \mathrm{CA} ; \mathrm{S}_{5}=1 / 2 \mathrm{~A}$ $+1 / 2 \mathrm{BC} ; \mathrm{S}_{6}=1 / 2 \mathrm{~A}+1 / 2 \mathrm{CA} \mathrm{e}_{7}=1 / 3 \mathrm{~A}+1 / 3 \mathrm{BC}+1 / 3 \mathrm{CA} ;$ onde $\mathrm{A}=$ areia; $\mathrm{BC}=$ bagaço de cana-de-açúcar; $\mathrm{CA}=$ casca de amendoim.

Médias seguidas por letras minúsculas distintas na coluna, e letras maiúsculas distintas na linha, diferem entre si pelo teste de Tukey a 5\% de probabilidade.

me de poros, quando comparado aos demais, o substrato $\mathrm{S}_{7}$ foi mais suscetível ao adensamento. Além disso, o substrato $\mathrm{S}_{7}$ apresentou a maior proporção volumétrica de bagaço de cana-deaçúcar (BC) e de casca de amendoim (CA), somando $67 \%$ do volume total, quando comparado aos substratos $\mathrm{S}_{5}(1 /$ $2 \mathrm{~A}+1 / 2 \mathrm{BC})$ e $_{6}(1 / 2 \mathrm{~A}+1 / 2 \mathrm{CA})$, com $50 \%$ de $\mathrm{BC}$ ou $\mathrm{CA}$, e aos substratos $\mathrm{S}_{2}(2 / 3 \mathrm{~A}+1 / 3 \mathrm{BC}), \mathrm{S}_{3}(2 / 3 \mathrm{~A}+1 / 3 \mathrm{CA})$

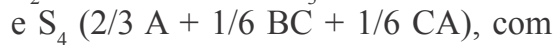
$33 \%$ de BC e/ou CA. Provavelmente, a decomposição também tenha ocorrido nos substratos com menor proporção volumétrica de $\mathrm{BC}$ e/ou CA, porém, até o final do segundo cultivo, a intensidade da decomposição não foi suficiente para promover alterações significativas no tamanho das partículas e, conseqüentemente, no tamanho dos poros formados, como observado para o substrato $\mathrm{S}_{7}$.

Concluindo, a reutilização do substrato composto por partes iguais de areia, bagaço de cana-de-açúcar e casca de amendoim promoveu aumento da densidade seca e do volume de água facilmente disponível, e redução da porosidade total, do espaço de aeração e do volume de água remanescente.

\section{AGRADECIMENTOS}

Ao CNPq, pela concessão da bolsa de doutorado.

\section{LITERATURA CITADA}

ABAD MB; NOGUERA PM; CARRIÓN CB. 2004. Los sustratos en los cultivos sin suelo. In: URRESTARAZU MG. (Ed.). Tratado de cultivo sin suelo. Madrid: Mundi-Prensa, p. 113-158. ANDRIOLO JL; DUARTE TS; LUDKE L; SKREBSKY EC. 1999. Caracterização e avaliação de substratos para o cultivo do tomateiro fora do solo. Horticultura Brasileira, 17: 215-219.

ANSORENA JM. 1994. Sustratos: propiedades y caracterizacion. Madrid: Mundi-Prensa, $172 \mathrm{p}$.

BACKES MA. 1988. Composto de lixo urbano como substrato para plantas ornamentais. $80 \mathrm{f}$. Dissertação (Mestrado em Fitotecnia) - Faculdade de Agronomia, Universidade Federal do Rio Grande do Sul, Porto Alegre.

BAEVRE OA. 1981. Chemical and physical properties of re-used peat for tomato. Acta Horticulturae, 126: 45-50.

BAEVRE OA; GUTTORMSEN G. 1984. Reuse of peat bags for tomatoes and cucumbers. Plant and Soil, 77: 207-214.

CARRIJO OA; VIDAL MC; REIS NVB; SOUZA RB; MAKISHIMA N. 2004. Produtividade do tomateiro em diferentes substratos e modelos de casas de vegetação. Horticultura Brasileira, 22: 05-09.

CELIKEL G; CAGLAR G. 1999. The effects of re-using different substrates on the yield and earliness of cucumber on autumn growing period. Acta Horticulturae, 492: 259-264.

DE BOODT M; VERDONCK O. 1972. The physical properties of the substrates in horticulture. Acta Horticulturae, 26: 37-44.

REIS M; INÁCIO H; ROSA A; CAÇO J; MONTEIRO. 2001. A Grape marc compost as an alternative growing media for greenhouse tomato. Acta Horticulturae, 554: 75-81.

RICHARDS D; LANE M; BEARDSELL DV. 1986.The influence of particle-size distribution in pinebark:sand:brown coal potting mixes on water supply aeration and plant growth. Scientia Horticulturae, 29: 1-14.

VERLODT H; ZOUAOUI M; SAIDANE A; WAELE N; HARBAOUI Y. 1985. Influence of reutilization during five years on the physical and chemical properties of a Posidonia oceanica (L.) Del. substrate and on the behavior of a tomate crop. Acta Horticulturae, 172: 157-173. 